\title{
The Tehran Eye Study: research design and eye examination protocol
}

\author{
Hassan Hashemi ${ }^{1,2}$, Akbar Fotouhi*3,2 and Kazem Mohammad ${ }^{3}$
} Address: ${ }^{1}$ Farabi Eye Hospital, School of Medicine, Tehran University of Medical Sciences, Tehran, Iran, ${ }^{2}$ Noor Vision Correction Center, Tehran,
Iran and ${ }^{3}$ Epidemiology and Biostatistics Department, School of Public Health, Tehran University of Medical Sciences, Tehran, Iran

Email: Hassan Hashemi - hhashemi@noorvision.com; Akbar Fotouhi* - afotouhi@sina.tums.ac.ir; Kazem Mohammad - mohamadk@sina.tums.ac.ir

* Corresponding author

Published: 15 July 2003

BMC Ophthalmology 2003, 3:8
Received: 28 January 2003

Accepted: 15 July 2003

This article is available from: http://www.biomedcentral.com/I47I-24I5/3/8

(C) 2003 Hashemi et al; licensee BioMed Central Ltd. This is an Open Access article: verbatim copying and redistribution of this article are permitted in all media for any purpose, provided this notice is preserved along with the article's original URL.

\begin{abstract}
Background: Visual impairment has a profound impact on society. The majority of visually impaired people live in developing countries, and since most disorders leading to visual impairment are preventable or curable, their control is a priority in these countries. Considering the complicated epidemiology of visual impairment and the wide variety of factors involved, region specific intervention strategies are required for every community. Therefore, providing appropriate data is one of the first steps in these communities, as it is in Iran. The objectives of this study are to describe the prevalence and causes of visual impairment in the population of Tehran city; the prevalence of refractive errors, lens opacity, ocular hypertension, and color blindness in this population, and also the familial aggregation of refractive errors, lens opacity, ocular hypertension, and color blindness within the study sample.
\end{abstract}

Methods Design: Through a population-based, cross-sectional study, a total of 5300 Tehran citizens will be selected from 160 clusters using a stratified cluster random sampling strategy. The eligible people will be enumerated through a door-to-door household survey in the selected clusters and will be invited. All participants will be transferred to a clinic for measurements of uncorrected, best corrected and presenting visual acuity; manifest, subjective and cycloplegic refraction; color vision test; Goldmann applanation tonometry; examination of the external eye, anterior segment, media, and fundus; and an interview about demographic characteristics and history of eye diseases, eye trauma, diabetes mellitus, high blood pressure, and ophthalmologic cares. The study design and eye examination protocol are described.

Conclusion: We expect that findings from the TES will show the status of visual problems and their causes in the community. This study can highlight the people who should be targeted by visual impairment prevention programs.

\section{Background}

Visual impairment is a global public health problem. Worldwide, an estimated 45 million people are blind, and an additional 135 million have severe visual impairment. $[1,2]$ The prevalence of blindness in developing countries is $10-40$ times higher than in developed countries, and close to three quarters of the world's blindness is either curable or preventable. The majority of blind people on earth reside in the developing nations of Africa, Asia, and Latin America. [3] There are several studies on the 
prevalence of visual impairment in the world [4-35] and Eastern Mediterranean countries. [3,7,31-33] Population-based data on frequency and causes of visual impairment are useful for identifying needs for treatment and rehabilitation services, planning and implementing blindness prevention programs, and determining research priorities for different populations.

There are very few published studies concerning this issue in the Iranian population. [36] Iran's National Health Survey provides data on visual impairment in this country. [37] These data, based on self-reporting, have some limitations. The goal of the present project is to describe the prevalence and causes of visual impairment in the population of Tehran city.

\section{Specific Objectives}

To determine the prevalence of visual impairment (low vision and blindness) in Tehran population.

To evaluate the determinants of visual impairment within the study sample.

To determine the prevalence of refractive errors, lens opacity, ocular hypertension and color blindness in Tehran population.

To describe the familial aggregation of refractive errors, lens opacity, ocular hypertension, and color blindness within the study sample.

\section{Methods/Design}

The Tehran Eye Study has been designed as a cross-sectional survey in the population of the urban area of Tehran city.

\section{Population and sampling strategy}

Tehran population

The Tehran region, which is a part of Tehran province, includes the cities of Tehran, Karaj, Savejbolagh, Shahryar, Shemiran, Rey, Eslamshahr and Varamin. On account of common borders and due to close economic and social links, this area is called "the Tehran region". These various cities became a collective entity in 1966, and their links have become more intensified since 1976. The 1996 census recorded Tehran region's population as 9.4 million, 6.8 million of which belonged to Tehran city alone.

The sampling frame is considered the population of the urban area of Tehran city. Table 1 shows the distribution of Tehran population and families in the 22 municipal districts according to the 1996 census. It is estimated that the present total population in this area is nearly 7.5 million, but there is no evidence suggesting any change in the distribution. So we will distribute our clusters on the basis of the population size of each district in 1996.

\section{Number of Households in Tehran City}

Census 1996 statistics showed that there were 1,660,219 households in Tehran city. Table 1 presents the number of households within each district of Tehran city. Households averaged 4.3 people in 1996.

\section{Sample Size}

For sample size calculation, the objective we have considered is to estimate the prevalence of variables with proportions as little as $0.02(\mathrm{P})$. On this assumption, for a $95 \%$ confidence interval $\left(\mathrm{Z}_{1-\alpha / 2}=1.96\right)$ and precision of 0.005 (d), the sample size is calculated as follows:

$\mathrm{n}=\frac{\mathrm{Z}_{(1-\alpha / 2)}^{2} \mathrm{P}(1-\mathrm{P})}{\mathrm{d}^{2}}=3010$

Considering a design effect of 1.5 and a response rate of $85 \%$, total sample size is calculated by the following equation:

$\mathrm{n}=3010 \times 1.5 \times 1 / 0.85 \approx 5300$.

\section{Sampling Plan}

This study follows a stratified cluster sampling strategy with proportional allocation within strata. The target population is all urban non-institutionalized citizens, of all ages, who reside in Tehran city in the year 2002. The stratification of the sample according to the 22 municipal districts of Tehran city is incorporated in the sampling process. Proportional to the number of households in the 22 districts (table 1 ), the appropriate number of clusters is assigned to each district (table 2). A total of 160 clusters are randomly selected based on blocks enumeration of the national census of 1996 by the Iranian Statistics Center. The decision about the number of clusters is based on total sample size; mean household members; and logistical facilities for subject enumeration, transport, and examination. For each cluster, a team of 2 interviewers (one male and one female) approaches the index household, which is specified through the aforementioned random selection of clusters, and continues the enumeration in 10 neighbor households in a systematic manner by proceeding round in a clock-wise direction. If more than one household inhabits a building, one will be randomly selected. They introduce themselves by presenting their identification cards. Then they describe the project to the present members of the households. At the end, all household members (people who have lived together in a housing unit for 6 months or more over the past year) are invited for a complete eye examination at Noor Vision Correction Center. They receive an invitation card in 
Table I: Distribution of Tehran population in the 22 municipal districts (1996 census).

\begin{tabular}{|c|c|c|c|c|}
\hline \multirow[b]{2}{*}{ District } & \multicolumn{2}{|c|}{ Family } & \multicolumn{2}{|c|}{ Population } \\
\hline & Number & Percent & Number & Percent \\
\hline I & 66,142 & 3.98 & 249,676 & 3.69 \\
\hline 2 & 120,333 & 7.25 & 458,089 & 6.78 \\
\hline 3 & 7I,746 & 4.32 & 259,019 & 3.83 \\
\hline 4 & $155,2 \mid 4$ & 9.35 & 663,166 & 9.81 \\
\hline 5 & 105,186 & 9.34 & 427,995 & 6.33 \\
\hline 6 & 60,063 & 3.62 & 220,331 & 3.26 \\
\hline 7 & 81,657 & 4.92 & 300,212 & 4.44 \\
\hline 8 & 89,103 & 5.37 & 336,474 & 4.98 \\
\hline 9 & 42,847 & 2.58 & 173,482 & 2.57 \\
\hline 10 & 75,064 & 4.52 & 282,308 & 4.18 \\
\hline II & 59,065 & 3.56 & 225,840 & 3.34 \\
\hline 12 & 48,579 & 2.92 & 189,625 & 2.81 \\
\hline 13 & 61,062 & 3.68 & 245,142 & 3.63 \\
\hline 14 & 98,140 & 5.91 & 394,611 & 5.84 \\
\hline 15 & 142,236 & 8.57 & 622,517 & 9.21 \\
\hline 16 & 71,269 & 4.29 & 298,410 & 4.42 \\
\hline 17 & 66,711 & 4.02 & 287,367 & 4.25 \\
\hline 18 & 64,241 & 3.87 & 296,243 & 4.38 \\
\hline 19 & 47,381 & 2.85 & 227,389 & 3.36 \\
\hline 20 & 80,835 & 4.87 & 356,079 & 5.27 \\
\hline 21 & 40,746 & 2.45 & 188,890 & 2.79 \\
\hline 22 & 12,599 & 0.76 & 56,020 & 0.83 \\
\hline Total & $1,660,219$ & 100 & $6,758,845$ & 100 \\
\hline
\end{tabular}

which the date and time of visit are clarified. The household members are informed that they will be transported to the clinic by the project staff. The enumeration teams approach clusters on Mondays and Tuesdays, and participants are examined on Thursdays and Fridays (formal weekend in Iran). Nine clusters are covered, and nearly 240 participants are visited weekly.

\section{Non-response}

Enumerated subjects who do not attend the examination process following the initial invitation will be contacted twice in subsequent weeks. Those who fail to appear even after the third invitation will be considered non-respondent.

\section{Examination Protocol}

The examination protocol includes lensometry; uncorrected, best corrected and presenting visual acuity measurements; manifest, subjective and cycloplegic refraction; color vision test; Goldmann applanation tonometry; examination of the external eye, anterior segment, media, and fundus; and an interview about demographic characteristics, history of eye diseases, eye trauma, diabetes mellitus, high blood pressure, and ophthalmologic cares (Figure 1).

\section{Visual Acuity and Refraction}

An optometrist determines the visual acuity by using a NIDEK chart projector (CP - 670 20/10-20/400; Nidek Co, Gamagori, Japan) with tumbling E letters at a distance of 4 meters. Best spectacle corrected and uncorrected visual acuity tests are performed separately for each eye (one eye at a time). Presenting visual acuity is measured with the participant's habitual distance correction. Lensometry is performed by an optometrist for those who use glasses. Visual acuity is recorded as the smallest line in which the patient can read the four letters correctly. If the person is unable to read the largest $\mathrm{E}$ letters in the chart $(20 / 400 \mathrm{E}$ letters) at 4 meters, then finger counting is done at 1 meter. The examiner stands one meter in front of the participant and asks if the participant can see his/her hand. The examiner slowly waves his/her hand and asks the participant if he/she can see what the hand is doing. If the participant is able to see the examiners hand moving, "hand motion" is recorded on the exam form. If the participant cannot see the examiner's hand, a penlight is held in front of the participant's eye and he/she is asked if he/ she can tell when the light is on. If the participant can correctly identify when the light is on, "light perception" is recorded on the exam form. If the participant is unable to see the light, "no light perception" is recorded. Care will 


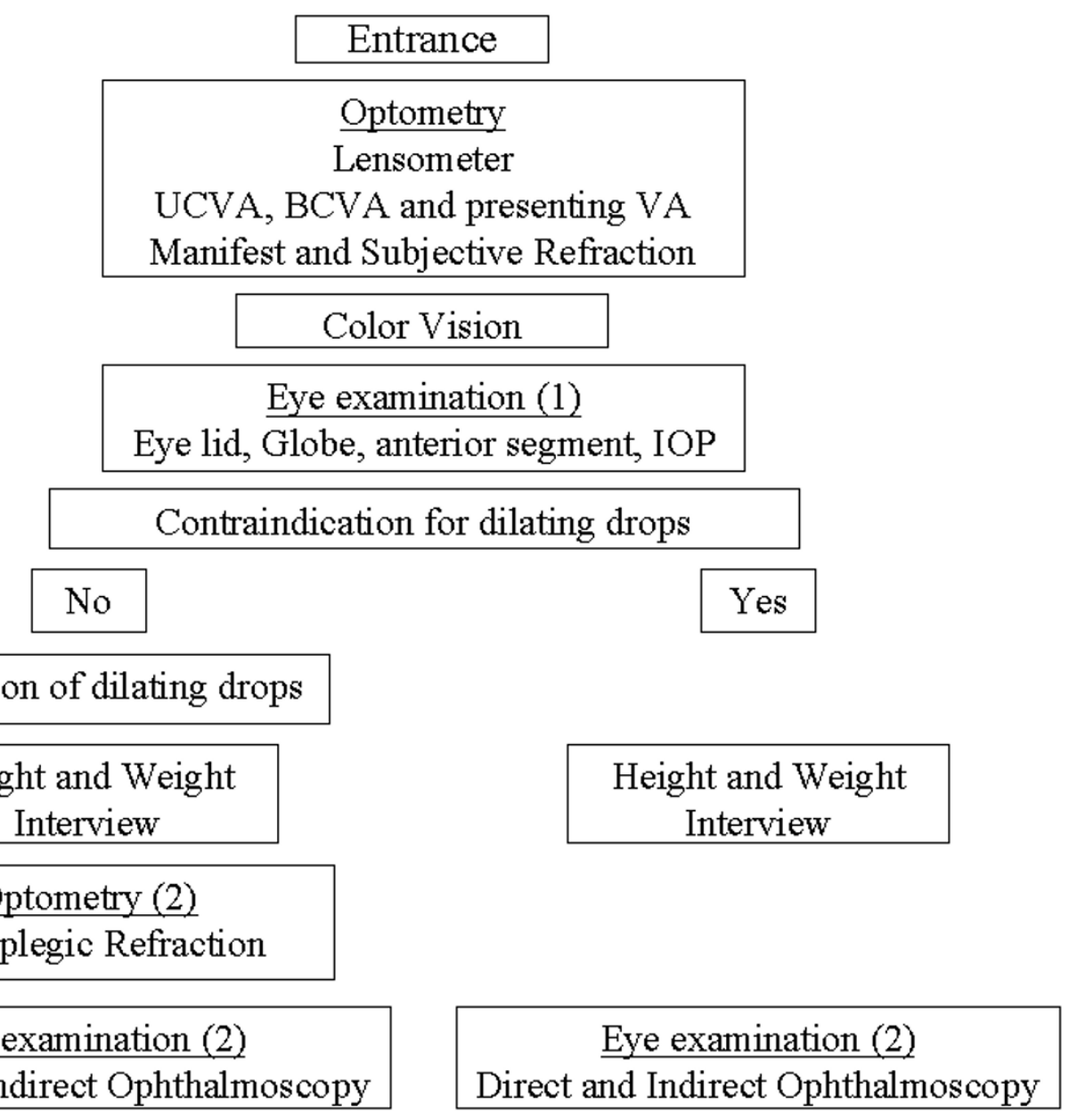

\section{Figure I}

Examination process flowchart. UCVA = uncorrected visual acuity; BCVA = best-corrected visual acuity; VA = visual acuity; $\mathrm{IOP}=$ intraocular pressure.

Table 2: Number of clusters in the 22 municipal districts of Tehran.

\begin{tabular}{lccc}
\hline District & Number of clusters & District & Number of clusters \\
\hline 1 & 6 & 12 & 5 \\
2 & 12 & 13 & 6 \\
3 & 7 & 14 & 9 \\
4 & 15 & 15 & 14 \\
5 & 10 & 16 & 7 \\
6 & 6 & 17 & 6 \\
7 & 8 & 18 & 6 \\
8 & 9 & 19 & 4 \\
9 & 4 & 20 & 8 \\
10 & 7 & 21 & 4 \\
11 & 6 & 22 & 1 \\
\hline
\end{tabular}


be taken to ensure that the unexamined eye is adequately covered with the palm or cloth and not pressed.

Refraction is done on all participants of five years of age and over using a Topcon automated refractometer (Topcon KR 8000, Topcon Corporation, Tokyo, Japan) by an optometrist. The optometrists act according to the instruction manual of Topcon KR 8000. Results from autorefraction are used as a starting point for a full subjective and manifest refraction. If autorefraction is not possible (especially due to a media opacity) manual manifest and subjective refraction is attempted. On the judgment of an ophthalmologist, if there is no contraindication, cycloplegic refraction is done. In this case, cyclopentolate $1 \%, 2$ drops are instilled 30 and 25 minutes before refraction. The participants are warned about the symptoms of cyclopentolate.

\section{Color vision test}

Using Farnsworth D-15 test, an optometrist tests color vision in all participants of at least 7 years of age. The Farnsworth D-15 test consists of 15 colored papers selected from the hue circle mounted in plastic caps. The participant is given the tray and allowed 2 minutes to arrange the hue in serial order according to their colors.

\section{Eye Examination}

Eyelid, globe, and anterior segment examination is performed at the first ophthalmologic visit. Presence of globe is recorded. In order to assess defective eyelid closure, the participant is asked to close the eyes. In cases of lagophthalmos, ectropion, loss of lid margin, etc, the lid may not come into apposition. Then the condition is specified.

For all participants of five years of age and over, a slitlamp examination is done (Topcon slitlamp, Topcon Corporation, Tokyo, Japan). The participant is seated comfortably at the slitlamp with his/her chin firmly on the chin rest and forehead against the headrest. The examiner then examines the anterior segment of each eye adjusting the beam width, magnification, and beam angle to achieve the best view of all the structures in the anterior segment. In viewing the cornea, the presence of arcus and the amount of involvement, any corneal scars, lesions, or abnormalities are recorded. The sclera, conjunctiva, and iris are all inspected for the presence of any lesions and abnormalities. The presence of pinguecula and pyterigia are recorded. A pyterigium is distinguished from a pingueculum if it crosses the limbus.

\section{Iris color determination}

Iris color is determined by viewing the participant's iris with the pupil undilated using a penlight. The color is compared to a color standards developed on the basis of the standards used in Beaver Dam Eye Study. Although the choices on the form are "gray or blue", "yellow or green", "light brown", "medium brown" and "dark", the amount of pigment in the iris is taken into account rather than the actual color. When an iris has more than one color, the grade is assigned based on the color which is $50 \%$ or more.

\section{Intraocular pressure}

The intraocular pressure (IOP) is measured using a Goldmann applanation tonometer. A drop of tetracain is instilled in each eye of the participant and tear is stained with fluorescein. The tonometer is swung into place on the slitlamp, the blue filter put into place, and the beam width opened to its fullest height. The beam angle should be about 45-60 degrees to the side of the tonometer and should illuminate the end of the prism head. The examiner should be aware of possible contact of the tonometer with the lids, lashes, or beard; all of which may cause a "high" reading. If IOP is $>30 \mathrm{mmHg}$, it is rechecked after dilation. If IOP is $>40 \mathrm{mmHg}$, the ophthalmologist prescribes appropriate treatment for the participant and informs him / her about the disease. Time of IOP measurement is recorded. The participant is asked whether he or she is currently taking medication for glaucoma. If the answer is affirmative, the drug is recorded.

\section{Angle assessment}

The angle of the anterior chamber is evaluated to determine the risk of occlusion upon dilation. The examiner assesses the angle by viewing it nasally and temporally with a narrow beam directed at an angle of about 45 degrees. If the ophthalmologist feels the chamber angle to be occludable, dilating drops are not administered. When the examiner is in doubt, another examiner will be consulted. If the chamber does not appear occludable, dilating drops are administered following the intraocular pressure test.

\section{Clinical lens opacities grading}

The ophthalmologist grades cortical and posterior subcapsular opacities, nuclear opalescence and color by visual comparison with a standard photograph (the Lens Opacities Classification System III, LOCS III) [38] through the biomicroscopic ophthalmic exam by a Topcon slitlamp (Topcon Corporation, Tokyo, Japan).

\section{Direct and Indirect ophthalmoscopy}

All participants of five years of age and over undergo a retinal exam first using direct ophthalmoscopy followed by indirect ophthalmoscopy. The retina is examined systematically to ensure that no lesions are passed over. The examiner inspects the optic disc assessing disc size, color, vascularity, and degree of cupping. The retinal exam proceeds systematically, not overlooking any lesions or abnormalities such as congenital anomalies, signs of early 
age-related maculopathy, retinitis pigmentosa, vascular retinopathy, Drusen, retinal detachment, and different types of diabetic retinopathy, which are recorded on the exam form if present.

\section{Definitions}

Visual impairment

The $10^{\text {th }}$ edition of the International Classification of Diseases (ICD10) defines visual impairment as a visual acuity of less than $6 / 18(20 / 60,0.3)$ in the better eye with the best correction. [39] Visual impairment is categorized to blindness and low vision. Blindness is a visual acuity of less than $3 / 60(20 / 400,0.05)$ in the better eye with the best correction. Low vision is defined as a best corrected visual acuity of less than $6 / 18(20 / 60,0.3)$ but not less than $3 / 60(20 / 400,0.05)$ in the better eye. The cause of visual impairment is identified by an ophthalmologist. Using the best judgment, the ophthalmologist will determine one cause for each eye thought to be the principal cause in either eye. When multiple disorders are present, the ophthalmologist attempts to identify the disorder causing the greatest limitation of vision. If there are any other contributory causes, the ophthalmologist specifies that as a second cause. In cases with different causes of visual reduction in the patient's two eyes, the diagnosis in the less affected eye is used. Cataract is considered the main cause of severe low vision if the fundus is obscured by lens changes, or if no evident fundus abnormalities are observed in eyes with significant cataract. Regarding the results of exams, the ophthalmologist specifies whether the participant needs any action and specifies the type of needed action.

\section{Observers Training and quality assurance}

All observers (including enumerators, optometrists and ophthalmologists) take part in a comprehensive training course, which has been developed for conducting the protocol. The course includes a full spectrum of education to ensure that all investigators have a broad based knowledge of the study process, study purposes, data forms, and technical skills needed to conduct the protocol in a scientifically sound manner. All staff must complete the training course prior to getting involved in the study. The program director and manager directly supervise the staff training process.

All observers receive regular quality control visits from the project manager who checks their performance. In addition, the data are reviewed periodically, and feedback is given to the observers weekly.

\section{Pilot and reliability study}

In order to assess the protocol, sampling process, participants' transport, data forms, equipment, and interobserver agreement, a pilot study is done one month before starting the study. The pilot study is done on 3 clusters in different districts outside those selected for the main study. To determine the reliability coefficient for visual acuity and refraction, the procedures are repeated twice by two series of observers. The agreement rates are calculated.

\section{Humanity and ethics}

The study is approved by the Research and Ethics Committee of Noor Vision Correction Center and Ethics Committee of the National Research Center for Medical Sciences. All subjects included in this study will be informed about the project and the procedures in their native language before being enrolled. They will be informed that their participation is entirely voluntary and they may decide to withdraw from the study at any time. The participants' agreement for examination will be obtained verbally. The confidentiality of all study participants will be protected in accordance with a good epidemiological practice.

\section{Data handling and statistical analysis \\ Data entry}

During the enumeration process, as data on each household is completed, a supervisor will do a quick check to see that all data is collected properly. Similarly, during the eye examination at the clinic, as each person completes the eye exam, the supervisor checks all data forms to ensure that all relevant data has been collected. Missing data and mistakes are rectified after consulting the concerned person.

\section{Data editing}

During data entry, the forms will be checked for completeness and consistency by the data entry software. If the forms are not filled in completely, the concerned person will be consulted to fill in the missing data or clarify an inconsistent data. All changes and coding will be made in ink by crossing out the original data and recording the new data beside it. It will be signed and dated by the person making the changes. Overwriting will be avoided.

\section{Statistical analysis}

In calculating standard errors and the 95\% confidence interval for categorical and continuous variables, the cluster sampling design is taken into account and adjusted for. [40] In addition to descriptive analyses, odds ratios are calculated with multivariate logistic regression in order to control potential confounding variables, and account for cluster design effects.

A familial association of qualitative variables may be assessed by an odds ratio (OR) [41] that, for any pair $j$ and $\mathrm{k}$ of individuals in a family, is defined as the odds of person $\mathrm{j}$ having an outcome given person $\mathrm{k}$ has the same outcome divided by the odds of person $\mathrm{j}$ having the outcome 
given person $\mathrm{k}$ does not. To adjust for possible confounding variables, we will use the second-order generalized estimating equations (GEE2) approach to logistic regression that simultaneously models the risk of a person having myopia and the familial associations. [41,42] In order to assess the degree of familial association for quantitative variables (e.g., spherical equivalent and cylindrical power) we computed Pearson correlation coefficients for all possible pairs of siblings. All analyses will be done using STATA statistical software, version 6.0 (STATA Corporation, TX).

\section{Discussion}

This study protocol describes the study design and eye examination of a cross-sectional population-based study in Tehran population. We expect that findings from the TES will show the status of visual problems and their causes in the community. This study will highlight the people who should be targeted by intervention programs for prevention of visual impairment.

\section{List of abbreviations used}

$\mathrm{VA}=$ visual acuity

UCVA = uncorrected visual acuity

$\mathrm{BCVA}=$ best corrected visual acuity

IOP = intraocular pressure

ICD10 $=$ The International Classification of Diseases, $10^{\text {th }}$ edition

LOCS III = The Lens Opacities Classification System III

\section{Competing interests}

None declared.

\section{Authors' contributions}

$\mathrm{HH}$, the director of the project, participated in the design of the study and the examination protocol, and he will supervise the examination process. AF, the manager of the project, participated in the design of the study and will coordinate the study. He drafted the manuscript and will participate in the statistical analyses. KM participated in the design of the study and will participate in the statistical analyses. All authors have read and approved the final manuscript.

\section{Acknowledgements}

Special thanks are given to Dr Ronald Klein, the Beaver Dam Eye Study; Dr Rupert Bourne, the National Blindness and Low Vision Prevalence Survey of Bangladesh; Dr Leon Ellwein and Dr Praveen K Nirmalan, the Tirunelveli eye survey; Dr Montserrat Martin-Baranera, Survey of blindness in Bioko, Equatorial Guinea, all who kindly have provided us the protocols and data forms of their studies. We also thank Dr Kamran Hojat Jalali and Dr Azam Alimardani for their helps in preparation of the eye examination protocol.

This project is supported in part by Noor Vision Correction Center, and a grant from the Iranian National Research Center for Medical Sciences. The selection of clusters is done based on blocks enumeration of the national census of 1996 by the Iranian Statistics Center.

\section{References}

I. Thylefors B: A mission for vision Lancet 1999, 354(SuppI):SIV44.

2. Thylefors B, Negrel AD, Pararajasegaram R and Dadzie KY: Global data on blindness Bull World Health Organ 1995, 73:115-121.

3. Tabbara KF: Blindness in the eastern Mediterranean countries BrJ Ophthalmol 200I, 85:77I-777.

4. Klein R, Klein BEK, Linton KLP and De Mets DL: The Beaver Dam Eye Study: visual acuity Ophthalmology I99|, 98:1310-1315.

5. Attebo K, Mitchell $P$ and Smith W: Visual acuity and the causes of visual loss in Australia. The Blue Mountains Eye Study Ophthalmology 1996, 103:357-364.

6. Rahmani B, Tielsch JM, Katz J, Gottsch J, Quigley H, Javitt J and Sommer $A$ : The cause-specific prevalence of visual impairment in an urban population. The Baltimore Eye Survey Ophthalmology 1996, 103:172I-1726.

7. Mansour AM, Kassak K, Chaya M, Hourani T, Sibai A and Alameddine $M N$ : National survey of blindness and low vision in Lebanon Br J Ophthalmol 1997, 81:905-906.

8. Rubin GS, West SK, Munoz B, Bandeen-Roche K, Zeger S, Schein O and Fried LP: A comprehensive of visual impairment in a population of older assessment The SEE Study. Salisbury Eye Evaluation Project. Americans Invest Ophthalmol Vis Sci 1997, 38:557-568.

9. Taylor HR, Livingston PM, Stanislavsky YL and McCarty CA: Visual impairment in Australia: distance visual acuity, near vision, and visual field findings of the Melbourne Visual Impairment Project Am J Ophthalmol 1997, I 23:328-337.

10. Klaver CCW, Wolfs RCW, Vingerling JR, Hofman A and de Jong PT: Age-specific prevalence and causes of blindness and visual impairment in an older population: The Rotterdam Study Arch Ophthalmol 1998, I 16:653-658.

II. Lee DJ, Gomez-Marin O and Lam BL: Prevalence of uncorrected binocular distance visual acuity in Hispanic and non-Hispanic adults Ophthalmology 1998, 105:552-560.

12. Zhao J, Jia L, Sui R and Ellwein LB: Prevalence of blindness and cataract surgery in Shunyi county, China Am J Ophthalmol I998, | 26:506-5|4.

13. Pokharel GP, Regmi G, Shrestha SK, Negrel AD and Ellwein LB: Prevalence of blindness and cataract surgery in Nepal $\mathrm{Br} J$ Ophthalmol 1998, 82:600-605.

14. Munier A, Gunning T, Kenny D and O'Keefe M: Causes of blindness in the adult population of the Republic of Ireland $\mathrm{Br} J$ Ophthalmol 1998, 82:630-633.

15. See JLS, Wong TY and Yeo KT: Trends in the Pattern of Blindness and Major Ocular Diseases in Singapore and Asia Ann Acad Med Singapore 1998, 27:540-546.

16. He M, Xu J, Li S, Wu K, Munoz SR and Ellwein LB: A survey of blindness and cataract surgery in Doumen County, China Ophthalmology 1999, 106:1602-1608.

17. Weih LM, VanNewkirk MR, McCarty CA and Taylor HR: Age-specific causes of bilateral visual impairment Arch Ophthalmol 2000, I I 8:264-269.

18. Van der Pols JC, Bates CJ, McGraw PV, Thompson JR, Reacher M, Prentice $A$ and Finch $S$ : Visual acuity measurements in a national sample of British elderly people $\mathrm{Br} J$ Ophthalmol 2000, 84:165-170.

19. Murthy GVS, Gupta S, Ellwein LB, Munoz SR, Bachani D and Dada VK: A population-based eye survey of older adults in a rural district of Rajasthan Ophthalmology 200I, 108:679-685.

20. VanNewkirk MR, Weih LA, McCarty CA and Taylor HR: Cause-specific Prevalence of Bilateral Visual Impairment in Victoria, Australia Ophthalmology 200I, 108:960-967.

21. Hyman L, Wu SY, Connell AMS, Schachat A, Nemesure B, Hennis A and Leske MC: Prevalence and Causes of Visual Impairment in the Barbados Eye Study Ophthalmology 200I, 108:175I-I756. 
22. Buch H, Vinding $T$ and Nielsen NV: Prevalence and Causes of Visual Impairment According to World Health Organization and United States Criteria in an Aged, Urban Scandinavian Population, The Copenhagen City Eye Study Ophthalmology 200I, 1 08:2347-2357.

23. Michon JJ, Lau J, Chan WS and Ellwein LB: Prevalence of visual impairment, blindness, and cataract surgery in the Hong Kong elderly $\mathrm{Br} J$ Ophthalmol 2002, 86: I33-139.

24. Moser CL, Martin-Baranera M and Vega F: Survey of blindness and visual impairment in Bioko, Equatorial Guinea $\mathrm{Br} J$ Ophthalmol 2002, 86:257-260.

25. Dandona R, Dandona L, Srinivas M, Giridhar P, Prasad MN, Vilas K McCarty and Rao GN: Moderate visual impairment in India: the Andhra Pradesh Eye Disease Study Br J Ophthalmol 2002, 86:373-377.

26. Munoz $B$ and West SK: Blindness and visual impairment in the Americas and the Caribbean Br J Ophthalmol 2002, 86:498-504.

27. Nirmalan PK, Thulasiraj RD, Maneksha V, Rahmathullah R, Ramakrishnan R, Padmavathi A, Munoz SR and Ellwein LB: A population based eye survey of older adults in Tirunelveli district of south India: blindness, cataract surgery, and visual outcomes Br J Ophthalmol 2002, 86:505-5I2

28. Kocur I and Resnikoff $S$ : Visual impairment and blindness in Europe and their prevention $\mathrm{BrJ}$ Ophthalmol 2002, 86:716-722.

29. Evans JR, Fletcher AE, Wormald RPL, Siu-Woon Ng E, Stirling $S$, Smeeth L, Breeze E, Bulpitt CJ, Nunes M, Jones D and Tulloch A: Prevalence of visual impairment in people aged 75 years and older in Britain: results from the MRC trial of assessment and management of older people in the community $\mathrm{Br} J$ Ophthalmol 2002, 86:795-800.

30. Zainal M, Ismail SM, Ropilah AR, Elias H, Arumugam G, Alias D, Fathilah J, Lim TO, Ding LM and Goh PP: Prevalence of blindness and low vision in Malaysian population: results from the National Eye Survey $1996 \mathrm{Br}$ J Ophthalmol 2002, 86:95I-956.

31. Khandekar R, Mohammed AJ, Negrel AD and AI Riyami A: The prevalence and causes of blindness in the Sultanate of Oman: the Oman Eye Study (OES) Br J Ophthalmol 2002, 86:957-962.

32. Amansakhatov S, Volokhovskaya ZP, Afanasyeva AN and Limburg $\mathrm{H}$ : Cataract blindness in Turkmenistan: results of a national survey $\mathrm{Br} J$ Ophthalmol 2002, 86: I207-I2IO.

33. Rodriguez J, Sanchez R, Munoz B, West SK, Broman A, Snyder RW, Klein R and Quigley $\mathrm{H}$ : Causes of Blindness and Visual Impairment in a Population-based Sample of U. S. Hispanics Ophthalmology 2002, 109:737-743.

34. Bourne RRA, Dineen B, Ali SM, Noorul Hug DM and Johnson G]: The national blindness and low vision prevalence of Bangladesh research design, eye examination methodology and results of pilot study Ophthalmic Epidemiol 2002, 9: I |9-132.

35. Thulasiraj RD, Rahmathulla R, Saraswati A, Selvaraj S and Ellwein LB The Sivaganga Eye Survey: I. Blindness and cataract surgery Ophthalmic Epidemiol 2002, 9:299-3I2

36. Mohammad K, Joghatai MT, Syiadati S and Rahgozar M: A national survey of visual disturbances In Iran J Medical Council of IRI 200I, 1 9:203-209.

37. Noorbala AA and Mohammad K: National Health Survey of Iran The National Research Center for Medical Sciences 200I.

38. Chylack LT Jr, Wolfe JK, Singer DM, Leske MC, Bullimore MA, Bailey IL, Friend J, McCarthy D and Wu SY: The Lens Opacities Classification System III. The Longitudinal Study of Cataract Study Group Arch Ophthalmol 1993, I I I:83 |-836.

39. World Health Organization: International Statistical Classification of Diseases and Related Health Problems Geneva 1992.

40. Bennett S, Woods T, Liyanage WM and Smith DL: A simplified method for cluster sample surveys of health in developing countries World Health Statistics Quarterly I99I, 44:98-106.

41. Liang KY and Beaty TH: Measuring familial aggregation by using odds-ratio regression models Genet Epidemiol 1991, 8:361-370.

42. Qaqish BF and Liang KY: Marginal models for correlated binary responses with multiple classes and multiple levels of nesting Biometrics 1992, 48:939-950.

\section{Pre-publication history}

The pre-publication history for this paper can be accessed here: http://www.biomedcentral.com/1471-2415/3/8/prepub

Publish with BioMed Central and every scientist can read your work free of charge

"BioMed Central will be the most significant development for disseminating the results of biomedical research in our lifetime. "

Sir Paul Nurse, Cancer Research UK

Your research papers will be:

- available free of charge to the entire biomedical community

- peer reviewed and published immediately upon acceptance

- cited in PubMed and archived on PubMed Central

- yours - you keep the copyright 